\title{
Comparison of Diagnostic E fficacy of Visual Inspection of Cervix with Acetic Acid and Pap Smear for Prevention of Cervical Cancer: Is VIA Superseding Pap Smear?
}

\author{
${ }^{1}$ Kamal Patil, ${ }^{2}$ G Durdi, ${ }^{3}$ KS Lakshmi, ${ }^{1}$ MK Swamy \\ ${ }^{1}$ Professor, Department of Obstetrics and Gynecology, Jawaharlal Nehru Medical College, Belgaum, Karnataka, India \\ ${ }^{2}$ Associate Professor, Department of Obstetrics and Gynecology, Jawaharlal Nehru Medical College, Belgaum, Karnataka, India \\ ${ }^{3}$ Resident, Department of Obstetrics and Gynecology, Jawaharlal Nehru Medical College, Belgaum, Karnataka, India
}

Correspondence: Kamal Patil, Professor, Department of Obstetrics and Gynecology, Jawaharlal Nehru Medical College, Belgaum590010, Karnataka, India, Phone: 0831-2472510, Fax: 0831-2470759, e-mail: kamalpatil1967@yahoo.co.in

\section{ABSTRACT}

Objectives: To estimate diagnostic efficacy of visual inspection with acetic acid (VIA) in comparison to Pap smear. Methods: This cross-sectional study was carried over a period of 24 months on 200 women attending colposcopy clinic at KLES Dr Prabhakar Kore Hospital and Medical Research Center, Belgaum, India. All women enrolled in study underwent Pap smear, VIA, colposcopy and biopsy. The sensitivity, specificity, PPV, NPV, false-positive rate and false-negative rate were calculated for VIA, Pap smear and colposcopy with biopsy as the reference standard.

Results: In our study, sensitivity and specificity of VIA were found to be $86.95 \%$ and $72.51 \%$ respectively, and that of Pap smear $37.68 \%$ and $92.36 \%$ respectively. Colposcopy showed higher sensitivity (94.20\%) and specificity (94.65\%).

Conclusion: VIA is a suitable primary screening procedure alternative to Pap smear as it has high sensitivity and negative predictive value. Keywords: Cervical cancer screening, Visual inspection with acetic acid (VIA), Pap smear, Low-grade squamous intraepithelial lesion, High-grade squamous intraepithelial lesion.

\section{INTRODUCTION}

In developed countries, initiation and sustenance of cervical cytology programs have resulted in large decline in cervical cancer incidence and mortality over the last 40 to 50 years. ${ }^{1}$

Though Pap smear has been used as screening method conventionally in India, studies have shown that it has low sensitivity, moreover there is a need for trained personnel, laboratory infrastructure and need for compliance with the follow-up. ${ }^{2,3}$ Hence, alternative strategies, like VIA, are being investigated.

VIA is simple, inexpensive and easy to carry out in large population, does not require any laboratory back up and can be performed reliably by trained paramedical workers and doctors. It neither requires second person for interpretation of result nor second visit by the patient to collect report.

In India, and as well as in other developing countries, VIA has been extensively investigated ${ }^{2-6}$ and the results have shown that sensitivity of VIA was higher than that of Pap smear. ${ }^{2,5,6}$ In most of the studies, reference standard was used only in screenpositive women. ${ }^{2,5,6}$ Estimates of accuracy from cross-sectional studies will suffer from verification bias if the reference standard for the final diagnosis is applied in different proportions of screen-positive and screen-negative subjects. ${ }^{7}$
The present study was undertaken to evaluate the diagnostic efficacy of VIA in comparison to Pap smear and reference standard was used for all participants for estimating the accuracy of screening tests.

\section{MATERIALS AND METHODS}

This was a cross-sectional study carried out in the colposcopy clinic at KLES Dr Prabhakar Kore Hospital and Medical Research Center, Belgaum, from 1st November 2006 to 31st October 2008. The study was approved by the institutional research and ethics committee.

Around 200, referred to colposcopy clinic with recurrent episodes of white discharge, postcoital bleeding, intermenstrual bleeding, postmenopausal bleeding and suspicious look of cervix on per speculum examination, were included in the study. Women with active infection of lower genital tract were treated with appropriate medication and then called for participation in the study. Women with active vaginal bleeding, pregnancy and those with frank growth on cervix were excluded from the study. Written and informed consent was obtained from all participants after a brief explanation of the procedure. 
After obtaining history, per speculum examination was performed followed by Pap smear with Ayre's spatula and cytobrush. Later visual inspection with acetic acid (VIA) using $5 \%$ acetic acid was done by a physician who was trained to perform VIA of cervix. VIA results were interpreted 1 minute after application of acetic acid using halogen bulb (100 watt). All the participants underwent colposcopy irrespective of VIA results. Colposcopy was performed by a colposcopist blinded to the VIA results. Biopsy was taken from abnormal areas under colposcopy guidance. When the colposcopy did not detect any abnormal area, four-quadrant biopsy was obtained from squamo columnar junction. Endocervical curettage was performed in women with unsatisfactory colposcopy.

The results of Pap smear were reported according to the 2001 Bethesda system. ${ }^{8}$ The result of Pap smear was considered positive, if it revealed low-grade squamous intraepithelial lesion (LSIL), high-grade squamous intraepithelial lesion (HSIL), carcinoma in situ (CIS), atypical endocervical cells (AGUS) or invasive cancer. The results of VIA were interpreted as VIA positive or VIA negative using International Agency for Research on Cancer (IARC) criteria. ${ }^{9}$ Colposcopy diagnosis was made based on modified Reid colposcopic index (RCI). ${ }^{8}$ Colposcopy was considered positive, if it revealed LSIL and above.

The reference standard for defining true disease status was colposcopy-guided biopsy or four-quadrant biopsy when colposcopy was normal so as to avoid verification bias. True disease was defined as histologically confirmed LSIL and above.

The sensitivity, specificity, positive predictive value (PPV), negative predictive value (NPV), false positive rate and false negative rate were calculated for pap smear, VIA and colposcopy with biopsy as reference standard. 95\% confidence interval (CI) was calculated for sensitivity, specificity, PPV and NPV.

\section{RESULTS}

Out of 200 women enrolled, maximum number of women were found to be in the age group 30 to 40 years (41\%). Mean age was 35 years. Majority of the study group were para two (46\%) and para three (34.5\%). The mean parity was two. The presenting complaint was recurrent episodes of white discharge in 120 women (60\%), postcoital bleeding in eight women (4\%), intermenstrual bleeding in two (1\%) women, and postmenopausal bleeding in 15 (7.5\%) women. Of the total study population, 112 (56\%) had suspicious looking cervix.

On VIA, 96 (48\%) women were found to have a positive result. Pap smear was normal in 11 women, 153 out of 200 women had inflammation and 36 had abnormal Pap smear. Among 36 women with abnormal Pap smear, there were 19 LSIL, 15 HSIL, one case of invasive carcinoma and one case of AGUS. On colposcopy, 70 women were found to have positive result. Among them there were $33 \mathrm{LSIL}, 33 \mathrm{HSIL}$ and four cases of invasive carcinoma.
On biopsy, true disease was confirmed in 69 cases out of 200 accounting for $34.5 \%$. It includes 33 LSIL, 27 HSIL and nine invasive carcinomas. Out of nine invasive carcinomas, none had visible growth on per speculum examination.

Correlation of VIA, Pap smear and colposcopy with biopsy results are shown in Table 1 to 3 . Out of 33 biopsy proven LSIL, 29 were detected by VIA, however, Pap smear could pick-up only seven cases. Colposcopy could diagnose 31 out of 33 biopsy proven LSIL cases. Out of 27 biopsy proven HSIL, 22 were detected by VIA, but Pap smear missed 13 cases and could pick-up only nine cases. Colposcopy detected 23 out of 27 biopsy proven HSIL. All the nine cases of biopsy proven invasive carcinoma were positive on VIA, on Pap smear four were reported as LSIL, four as HSIL and one as invasive carcinoma, and colposcopy reported five as HSIL and four as invasive carcinoma.

The sensitivity, specificity, positive predictive value and negative predictive value of the individual tests are presented in Table 4 with their $95 \%$ confidence interval. Sensitivity and negative predictive value of VIA was more than that of Pap smear, however, specificity of Pap smear was higher compared to VIA.

Table 1 Correlation between VIA and biopsy

\begin{tabular}{|lcccccc|}
\hline VIA & \multicolumn{4}{c}{ Biopsy } & Total \\
\cline { 2 - 5 } & Normal & Cervicitis & LSIL & HSIL & Malignancy & \\
\hline Positive & - & 36 & 29 & 22 & 09 & 96 \\
Negative & 05 & 90 & 04 & 05 & 00 & 104 \\
\hline Total & 05 & 126 & 33 & 27 & 09 & 200 \\
\hline
\end{tabular}

Table 2 Correlation between Pap smear and biopsy

\begin{tabular}{|lcrrrrrr} 
Pap smear & \multicolumn{5}{c}{ Biopsy } & Total \\
\cline { 2 - 6 } & Normal & Cervicitis & LSIL & HSIL & Malignancy & \\
\hline Normal & 02 & 08 & 01 & - & - & 11 \\
Inflammatory & 03 & 107 & 25 & 18 & - & 153 \\
LSIL & - & 06 & 06 & 03 & 04 & 19 \\
HSIL & - & 04 & 01 & 06 & 04 & 15 \\
Malignancy & - & - & - & - & 01 & 01 \\
AGUS & - & 01 & - & - & - & 01 \\
\hline Total & 05 & 126 & 33 & 27 & 09 & 200
\end{tabular}

Table 3 Correlation between colposcopy and biopsy

\begin{tabular}{|c|c|c|c|c|c|c|}
\hline \multirow[t]{2}{*}{ Colposcopy } & \multicolumn{5}{|c|}{ Biopsy } & \multirow[t]{2}{*}{ Total } \\
\hline & Normal & Cervicitis & LSIL & HSIL & Malignancy & \\
\hline Normal & 03 & 11 & - & - & - & 14 \\
\hline $\begin{array}{l}\text { Infection/ } \\
\text { metaplasia/ } \\
\text { erosion }\end{array}$ & 02 & 100 & 02 & 02 & - & 106 \\
\hline LSIL & - & 06 & 24 & 03 & - & 33 \\
\hline HSIL & - & 01 & 07 & 20 & 05 & 33 \\
\hline Malignancy & - & - & - & - & 04 & 04 \\
\hline Unsatisfactor & ry - & 08 & - & 02 & - & 10 \\
\hline Total & 05 & 126 & 33 & 27 & 09 & 200 \\
\hline
\end{tabular}




\begin{tabular}{|clll|}
\hline Table 4 & \multicolumn{3}{|c}{ Diagnostic efficacy of tests and their 95\% confidence } \\
& interval & & \\
\hline & VIA & Pap smear & Colposcopy \\
\hline Sensitivity & $86.95 \%$ & $37.68 \%$ & $94.20 \%$ \\
& $(79.01-94.90)$ & $(26.25-49.12)$ & $(88.69-99.72)$ \\
Specificity & $72.51 \%$ & $92.36 \%$ & $94.65 \%$ \\
& $(64.87-80.16)$ & $(87.82-96.91)$ & $(90.81-98.51)$ \\
PPV & $62.50 \%$ & $72.22 \%$ & $90.27 \%$ \\
& $(52.82-72.18)$ & $(57.59-86.85)$ & $(83.43-97.12)$ \\
NPV & $91.34 \%$ & $73.78 \%$ & $96.87 \%$ \\
& $(85.94-96.75)$ & $(67.05-80.51)$ & $(93.86-99.89)$ \\
\hline
\end{tabular}

VIA showed higher false positive rate of $27.48 \%$ much higher to that of Pap smear (7.63\%). Colposcopy had false positive rate of 5.3\%. False negative rate of VIA (13\%) was less compared to Pap smear (62.31\%). Colposcopy had false negative rate of $5.79 \%$.

\section{DISCUSSION}

More than $80 \%$ of all cervical cancer occurs in developing countries where as developed countries have experienced decline in incidence and mortality of disease. This disparity is attributed primarily to the differences in screening and treatment of precancerous lesions. ${ }^{10}$

Present study aimed at evaluating diagnostic efficacy of two screening procedures of VIA and Pap smear for detection of precancerous lesions. Our study involved 200 women referred to colposcopy clinic. The reference standard was used for all participants to avoid verification bias and all the tests were carried out at the same visit.

We had VIA positivity rate of $48 \%$. Various studies have shown VIA to be positive in 6.9 to $51 \%$ of cases. ${ }^{11-14}$ VIA positivity rate depends upon incidence of carcinoma of cervix in community, type of criteria used for interpretation of results and type of population screened. Our VIA positivity rate was significantly higher than that found in other studies and similar to study done by Bhatla $\mathrm{N}$ et al. ${ }^{14}$

The results from present study indicate that VIA is a promising method of cervical cancer screening as it showed higher sensitivity (86.92\%) compared to Pap smear (36.23\%). Majority of the studies shown that sensitivity of VIA was more than that of Pap smear, however, Pap smear had high specificity than VIA. ${ }^{2,5,6,14,15}$ Our findings were similar to the pooled analysis of eleven studies done in Africa and India, on cervical cancer screening tests. VIA showed sensitivity of 79 and $83 \%$ for $\mathrm{CIN}^{+}$and $\mathrm{CIN}^{+}$respectively. Sensitivity and specificity of Pap smear were 57 and 93\% respectively. ${ }^{15}$ However, in our study, LSIL and above were considered as true disease. Our study showed higher sensitivity of VIA, probably, because screening was performed by trained physician using halogen bulb (100 watt) and study done on a group of women with symptoms, such as recurrent white discharge, abnormal uterine bleeding and suspicious look of cervix, on per speculum examination.

On biopsy, 69 women were found to have true disease, out of these VIA missed only nine cases (4 LSIL and 5 HSIL) whereas Pap smear missed 44 cases (26 LSIL and 18 HSIL). Out of nine cases which were missed by VIA; colposcopy detected seven cases which include four cases of LSIL and three cases of HSIL.

VIA showed high negative predictive value of $91.34 \%$ in our study and this was similar to other studies. ${ }^{2,5}$ Since VIA gives immediate result and has high negative predictive value of woman with negative VIA result can be assured immediately that she is disease-free.

In our study, VIA had high false positive rate (27.48\%) because 36 cases of biopsy proven cervicitis were reported as positive on VIA. Out of these 36 cases, 30 were found to be disease free on colposcopy. Davis-Dao PA et al ${ }^{16}$ found that presence of cervicits may influence the accuracy of VIA results.

The sensitivity and specificity of colposcopy was 94.2 and $94.65 \%$ respectively. In our study, sensitivity of colposcopy was less compared to study done by Goel A et $\mathrm{al}^{2}$ because we obtained biopsy from all participants.

VIA can be used as a screening procedure as it is simple, inexpensive, gives result immediately and can be performed by trained paramedical workers. VIA can be recommended as a screening tool not only in rural areas but also in well-equipped hospitals. Noncytology based screen-and-treat approaches for cervical cancer prevention have been proposed in developing countries. ${ }^{17}$ However, this approach leads to overtreatment, if VIA alone is used as a screening procedure before proceeding with treatment.

We believe that all VIA positive women should be referred to colposcopy and treatment of preinvasive lesions can be performed during the same visit in hospital setting, which will certainly have favorable implications for the cost of screening.

Addition of HPV testing to VIA has been proposed to increase the specificity of VIA, thereby reducing the referral rates without compromising the sensitivity of the test. ${ }^{14}$ Study done by Shastri SS et al proposed that in settings where only infrequent screening is possible and it is not feasible to provide good quality cytology, the use of a parallel combination of both VIA and visual inspection with lugol's iodine (VILI) should be considered. ${ }^{18}$

\section{CONCLUSION}

VIA is a suitable primary screening procedure alternative to Pap smear as it has high sensitivity and negative predictive value. Women with positive VIA result should be subjected to colposcopy to avoid unnecessary treatment in disease free, as VIA has high false positive rate.

\section{REFERENCES}

1. Sankaranarayanan R, Madhukar AB, Rajkumar R. Effective screening programmes for cervical cancer in low and middleincome developing countries. Bull World Health Organ, Suppl 2001;79(10):954-62.

2. Goel A, Gandhi G, Batra S, Bhambani S, Zutshi V, Sachdeva P. Visual inspection of the cervix with acetic acid for cervical intraepithelial lesions. Int J Gynecol Obstet 2005;88:25-30. 
3. Saira AS, Batra S, Gandhi G, Zutshi V. Comparison of methods used for screening of cervical preinvasive lesions. J Obstet Gynecol Ind 2003;3:70-71.

4. Sankarnarayanan R, Esmy PO, Rajkumar R, Muwonge R, Swaminathan R, Shanthakumari S, et al. Effect of visual screening on cervical incidence and mortality in Tamil Nadu: A cluster-randomised trial. Lancet 2007;4:370(9585):398-406.

5. Doh AS, Nhele NN, Achu P, Essimbi F, Essame O, Nkegoum B. Visual inspection with acetic acid and cytology for cervical lesions in Cameroon. Int J Gynecol Obstet 2005;89:167-73.

6. Abdel-Hady ES, Emam M, Al-Gohary A, Hassan M, Farag MK, Abo-Elkheir M, et al. Screening of cervical carcinoma using visual inspection with acetic acid. Int J Gynecol Obstet 2006; 93(2):118-22.

7. Sankarnarayanan R, Gaffikin, Jacob M, Sellors J, Robles S. A critical assessment of screening methods for cervical neoplasia. Int J Gynecol Obstet 2005;89:S4-12.

8. John WS, Sankarnarayanan R. Colposcopy and treatment of cervical intraepithelial neoplasia: A begginners' manual: IARC Lyon 2003;15,128-129.

9. Sankarnarayanan R, Wesley RS. A practical manual on visual screening for cervical neoplasia. IARC Technical Publication, No 41. Lyon, France; IARC Press 2003;15-26.

10. Mahboobeh S, Diane S, Philip EC. Cervical cancer preventioncervical screening: Science in evolution. Obstet Gynecol Clin N Am 2007;34:739-60.

11. Jose J, Orlando M, Jorge H, Jose P, Javier M, Jorge R, et al. Visual inspection with acetic acid for cervical cancer screening outside of low-resource settings. Rev Panam Salud Publica/ Pan Am J Public Health 2005;17(1):1-5.
12. Sarian SO, Derchain SF, Naud P, Martins CR, Tatti S, Branca $\mathrm{M}$, et al. Evaluation of visual inspection with acetic acid (VIA), lugol's iodine (VILI), cervical cytology and HPV testing as cervical screening tools in Latin America. J Med Screen 2005; 12(3):142-49.

13. Sankarnarayanan R, Basu P, Wesley RS, Mahe C, Keita N, Mbalawa CG, et al. Accuracy of visual screening for cervical neoplasia: Results from an IARC multicentre study in India and Africa. Int J Cancer 2004;110:907-13.

14. Bhatla N, Mukhopadhyay A, Kriplani A, Pandey RM, Gravitt $\mathrm{PE}$, Shah KV, et al. Evaluation of adjunctive tests for cervical cancer screening in low resource settings. Indian J Cancer 2007; 44(2):51-55

15. Arbyn M, Sankarnarayanan R, Muwonge R, Keita N, Dolo A, Mbalawa CG, et al. Pooled analysis of the accuracy of five cervical cancer screening tests assessed in eleven studies in Africa and India. Int J Cancer 2008;123(1):153-60.

16. Davis-Dao CA, Cremer M, Felix J, Cortessis VK. Effect of cervicitis on visual inspection with acetic acid. J Low Genit Tract Dis 2008;12(4):282-86.

17. Denny L, Kuhn L, De Souza M, Pollack AE, Dupree W, Wright TC. Screen-and- treat approaches for cervical cancer prevention in low-resource settings: A randomized controlled trial. JAMA 2005;294(17):2173-81.

18. Shastri SS, Dinshaw K, Amin G, Goswami S, Patil S, Chinoy $\mathrm{R}$, et al. Concurrent evaluation of visual, cytological and HPV testing as screening methods for the early detection of cervical neoplasia in Mumbai, India. Bull Word Health Organ 2005;83(3):186-94. 